\title{
Concepções e concepções alternativas de média: Um estudo comparativo entre professores e alunos do Ensino Fundamental ${ }^{1}$
}

\section{Conception and alternative conception of mean: a comparative study among teachers and students from basic school}

\author{
Sandra Magina ${ }^{2}$ \\ Irene Cazorla ${ }^{3}$ \\ Verônica Gitirana ${ }^{4}$ \\ Gilda Guimarães ${ }^{5}$
}

\begin{abstract}
RESUMO
O presente estudo analisa concepções de estudantes e professores sobre média, tanto aquelas válidas quanto as alternativas, com validade para alguns casos. Um teste, em lápis e papel, composto de 3 questões foi elaborado e aplicado com 287 estudantes e professores brasileiros, dos quais: 54 eram alunos da $4^{\mathrm{a}}$ série e 47 eram da $5^{\mathrm{a}}$ série do ensino fundamental, 61 eram estudantes iniciantes e 82 eram estudantes concluintes do curso de pedagogia, e 43 eram professores das séries iniciais do ensino fundamental. $\mathrm{O}$ teste foi aplicado
\end{abstract}

1 Este artigo refere-se a um estudo realizado no âmbito do projeto "A matemática escolar: construção e transformação do saber matemático", financiado pelo Programa Nacional de Cooperação Acadêmica (Procad) Coordenação de Aperfeiçoamento de Pessoal de Nível Superior (Capes).

$2 \mathrm{PhD}$ pela Universidade de Londres e pós-doutora pela Universidade de Lisboa, é professora do Programa de Pós-Graduação em Educação Matemática da Pontifícia Universidade Católica de São Paulo (PUC/SP) - Brasil. E-mail: sandra@pucsp.br.

3 Doutora pela Universidade Estadual de Campinas (UNICAMP) e pós-doutora pela PUC/ SP, é professora da Universidade Estadual de Santa Cruz (UESC/BA) - Brasil. E-mail: icazorla@ uol.com.br.

$4 \mathrm{PhD}$ pela Universidade de Londres, é professora do Programa de Pós-graduação Educação Matemática e Tecnologia da Universidade Federal de Pernambuco (UFPE) - Brasil. E-mail: veronica. gitirana@gmail.com.

5 Doutora em Psicologia Cognitiva, é professora do Programa de Pós-graduação Educação Matemática e Tecnologia da Universidade Federal de Pernambuco (UFPE) - Brasil . E-mail: gilda@ufpe.br. 
de forma coletiva, em sala de aula. Excetuando-se o grupo de professores, todos os grupos tendem a confundir média com adição dos valores ou com o valor máximo. Os resultados mostram que, apesar da evolução no entendimento do conceito de média em relação ao nível de escolaridade, algumas confusões ainda são identificadas entre o grupo de professores.

Palavras-chave: Tópicos específicos da Educação; Letramento Estatístico; média aritmética; concepções errôneas; Estudo Diagnóstico.

\begin{abstract}
The present study aims to analyze students and teachers conceptions and misconceptions of average. A paper and pencil test, comprised of three questions, was designed. 287 Brazilian students and teachers undertook the test. They were: 54 pupils from 4 th grade, 47 from 5 th grade, 61 students beginning undergraduation on Pedagogy, 82 students finishing their undergraduation on Pedagogy and 43 elementary school teachers. The test was undertaken in a collective way. Excepting to the teachers group, all the groups tend to confuse average to addition of the values, or to the maximum value. The result points that, in spite of an evolution on the understanding of the concept of average according to the educational level of the groups, some misconceptions are found within the teachers' group.
\end{abstract}

Keywords: Specific topics on Education; statistics literacy; average; misconception; Diagnostic Study.

\title{
Letramento estatístico
}

O papel da escola na formação de cidadãos tem sido foco de preocupações de Educadores do Brasil e do Mundo. Resultados de avaliações de larga escala tal como o Program for International Student Assessment - PISA têm questionado o sistema educacional brasileiro. Este é um programa de avaliação comparada, cuja principal finalidade é produzir indicadores sobre a efetividade dos sistemas educacionais na formação de seus estudantes para desempenhar um papel ativo como cidadãos, na sociedade. Para tanto, em vez de limitar-se a conhecer quais conteúdos do currículo os estudantes aprenderam, o foco da avaliação está centrado em estabelecer se os estudantes, de 15 anos de idade, matriculados nas escolas, podem utilizar o que aprenderam em situações usuais da vida cotidiana. O PISA busca avaliar a capacidade dos estudantes de analisar, raciocinar e comunicar eficazmente quando enunciam, formulam e resolvem problemas matemáticos em uma variedade de domínios e situações relacionadas à vida cotidiana, definindo esse tipo de avaliação como "letramento matemático". 
A análise da natureza dos problemas que permeiam a vida cotidiana das pessoas mostra que, cada vez mais, tais problemas envolvem conceitos e procedimentos estatísticos como gráficos, tabelas, médias, dentre outros, que subsidiam a tomada de decisões das pessoas. Esse conhecimento sobre como interpretar e avaliar criticamente as informações estatísticas tem sido denominado por vários autores de letramento estatístico (GAL, 2002; WATSON; CALLINGHAM, 2003).

Para Gal (2002), um adulto que vive numa sociedade industrializada é considerado letrado em Estatística quando consegue interpretar e avaliar criticamente informações estatísticas, discutindo ou comunicando sua compreensão sobre as implicações dessas informações e das conclusões fornecidas. Nessa definição está implícito o conhecimento de conceitos e procedimentos básicos de Estatística, ou seja, as apresentações gráficas e tabulares, as medidas de resumo, as medidas de tendência central e de variabilidade e Watson e Callingham (2003) propõem diferentes níveis hierárquicos do letramento estatístico.

O reconhecimento da importância do papel da escola no desenvolvimento do pensamento estatístico e da competência estatística na formação para a cidadania fez com que a maioria dos países incluísse os conceitos elementares nos seus currículos da Educação Básica (BATANERO et al., 1994). O Brasil também aderiu a essa tendência e, através dos Parâmetros Curriculares Nacionais da Educação Básica, oficializou seu ensino ao incluir o bloco Tratamento da Informação como o quarto bloco de conteúdos na disciplina de Matemática, no Ensino Fundamental (BRASIL, 1997, 1998) e a Análise de Dados no Ensino Médio (BRASIL, 2002, 2006).

Contudo, o ensino de Estatística na Educação Básica ainda enfrenta grandes desafios. Para Batanero (2000), é um paradoxo exigir que o professor de Matemática ensine Estatística quando esse, no seu curso de graduação, não teve formação didática específica. Além disso, a pesquisa na Didática da Estatística está sendo iniciada e estamos apenas conhecendo as principais dificuldades dos estudantes, devendo esses resultados serem disponibilizados aos professores.

Nesse contexto, foi desenvolvida uma pesquisa comparativa que investigou as competências e concepções de alunos e professores primários, em relação à leitura de tabelas, gráficos e medidas de tendência central. Neste artigo apresentamos, apenas, os resultados em relação à compreensão do conceito de média.

\section{Conceito de média}

Segundo Pollatsek, Lima e Well (1981), a média aritmética não é só o conceito mais básico da Estatística e da ciência experimental, é também o mais 
utilizado na vida cotidiana das pessoas. Em geral, ao fazermos inferências tanto no campo acadêmico como na vida cotidiana, utilizamos a média ou a comparação entre médias.

A média fornece um indicador que pode ser interpretado como um valor típico e que pode representar, em certas circunstâncias, um conjunto de dados. Além disso, é a base para o cálculo de outras medidas tais como o desvio padrão, coeficiente de variação, de correlação, dentre outras.

Para dados não agrupados, a média simples é calculada como o quociente entre a soma de todos os valores da variável e o número de observações envolvidas nessa soma. Para dados ponderados ou agrupados, os valores da variável devem ser ponderados pelos seus respectivos pesos ou frequências - nesse caso, a média é chamada de ponderada, cujas notações matemáticas são mostradas a seguir:

Média simples $\quad \bar{X}=\frac{\sum_{i=1}^{n} X_{i}}{n} \quad$ Média ponderada $\quad \bar{X}=\frac{\sum_{i=1}^{n} f_{i} X_{i}}{n}$

onde $X_{i}$ são valores assumidos pela variável, $n$ é o número de dados e $f_{i}$ é o peso ou ponderação

O domínio do conceito de média está intimamente relacionado à compreensão de suas propriedades, que segundo Strauss e Bichler (1988) são:

a) a média está localizada entre os valores extremos (valor mínimo $\leqslant$ média $\leqslant$ valor máximo);

b) a soma dos desvios a partir da média é zero $(\Sigma(\mathrm{Xi}-$ média $)=0)$;

c) a média é influenciada por cada um e por todos os valores (média $=$ $\Sigma \mathrm{Xi} / \mathrm{n})$;

d) a média não necessariamente coincide com um dos valores que a compõem;

e) a média pode ser um número que não tem um correspondente na realidade física (por exemplo, o número médio de filhos por casal é de 2,3);

f) o cálculo da média leva em consideração todos os valores inclusive os nulos e os negativos;

g) a média é um valor representativo dos dados a partir dos quais ela foi calculada. Em termos espaciais, a média é o valor que está mais próximo de todos os valores.

Conhecer o algoritmo e calcular a média não é garantia de sua compreensão, como mostram os resultados de várias pesquisas na área (BATANERO 
et al., 1994). Uma das dificuldades diz respeito à interpretação da média envolvendo números inteiros, cujo resultado é um número decimal como, por exemplo, o número médio de filhos por casal ser igual a 2,3. Essa falta de compreensão passa, de um lado, no caso de crianças pequenas, pela incompreensão do número decimal como mostra o trabalho de Watson (1996) e Selva e Borba (2005) e, de outro, pela ausência de compreensão da relação entre duas grandezas: número total de filhos e número total de casais, como mostra Cazorla (2003), que trabalhou com 840 estudantes de cursos de graduação, em nível universitário. Nesses estudos, desde a educação infantil até o ensino superior, os estudantes não levaram em consideração duas propriedades da média: que essa não necessariamente coincide com um dos valores da variável e que pode não ter correspondência física na realidade.

Uma outra dificuldade diz respeito ao uso da média ponderada, como por exemplo, o problema formulado por Pollatsek e outros (1981). Eles solicitaram a 17 estudantes de Psicologia que calculassem o peso médio de dez pessoas num elevador, assim distribuídas: quatro mulheres pesando em média 120 libras e seis homens pesando em média 180 libras. A maioria dos estudantes utilizou a média simples de 120 e 180 . Esse resultado também foi encontrado na pesquisa de Cazorla (2003) que, além disso, encontrou que $44,5 \%$ de seus sujeitos se limitaram a somar os pesos das dez pessoas. A soma dos valores da variável no lugar da média é outra concepção (sem validade estatística) comumente encontrada nas respostas dos estudantes, o que permite inferir que esses não conhecem ou não levam em consideração que o valor da média deve estar entre os valores extremos.

\section{Procedimentos e método}

Para analisar as concepções de estudantes e professores que atuam nas séries iniciais do Ensino Fundamental na cidade de São Paulo - Brasil, foi desenhada uma pesquisa exploratória, a qual constou da aplicação de um instrumento diagnóstico. Participaram do estudo 287 sujeitos, divididos em cinco grupos distinto, sendo: grupo 1, composto por 54 alunos de $4^{\mathrm{a}}$ série (com média de idade de 10 anos); grupo 2, contendo 47 alunos da $5^{\text {a }}$ série (com média de idade de 11 anos); grupo 3, formado por 61 alunos iniciantes do curso de Pedagogia; grupo 4, com 82 alunos concluintes do curso de Pedagogia e; grupo 5, composto por 43 professores do ensino fundamental. $\mathrm{O}$ instrumento foi aplicado de forma coletiva em cada um dos grupos e resolvido individualmente. $\mathrm{O}$ instrumento utilizado foi um teste em lápis e papel, contendo sete atividades envolvendo 
leitura, interpretação e construção de tabelas e gráficos e o conceito de média. O presente trabalho refere-se apenas à análise das três atividades, as quais diziam respeito a atividades que envolveram o conteúdo de média aritmética simples, as quais estão abaixo apresentadas na figura 1 a seguir.

\section{FIGURA 1 - AS TRÊS QUESTÕES DE MÉDIA UTILIZADAS NO INSTRUMENTO}

Atividade 1 (A1): Letícia está pesquisando preços de salgadinhos para comprar mais barato e economizar sua mesada. Ela anotou preços de três lugares diferentes e descobriu que o preço médio destes salgadinhos é de $\mathrm{R} \$ 3,00$.

Assinale V (Verdadeiro) ou $\mathrm{F}$ (Falso) para os possíveis valores que Letícia encontrou:
a) ( ) 1,1,1 b) ( ) 1,3,5 c) ( ) 3,3,3
d) ( ) 1,3,6 e) ( ) 9,9,9 f) ( ) 1,2,6

Descreva como você pensou.

Atividade 2 (A2): O gráfico ao lado mostra o consumo mensal de água de uma família durante seis meses: Alguns alunos calcularam o consumo médio de água desta família durante os seis meses. Leia as conclusões de cada aluno:

- João disse que o consumo médio foi de $54 \mathrm{~m}^{3}$.

- Carolina disse que o consumo médio foi de $12 \mathrm{~m}^{3}$.

- Marcelo disse que o consumo médio foi de $9 \mathrm{~m}^{3}$.

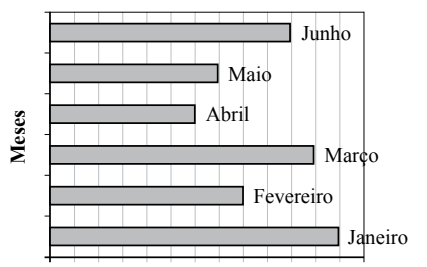

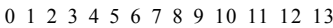
Consumo $\left(\mathbf{m}^{3}\right)$

Quem está certo?

Por quê?

Atividade 3 (A3): Observe atentamente o gráfico ao lado e responda:

Qual a região cuja média de assaltos por dia é a mais alta?

( ) Região Nordeste

( ) Região Sudeste

( ) Região Sul

( ) Todas

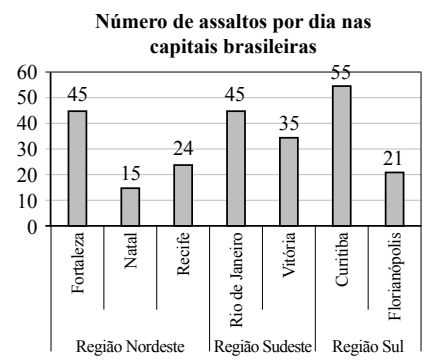

( ) Nenhuma 


\section{Resultados}

Procederemos com a análise dos resultados em duas etapas. Na primeira, trataremos os dados na perspectiva quantitativa. Assim consideraremos os percentuais de acertos quantitativos dos grupos nas três questões como um todo e, na sequência, em cada uma das questões. Na segunda parte buscaremos identificar a qualidade dos erros, procedendo assim com uma análise qualitativa dos resultados.

\section{Análise quantitativa dos resultados}

A Figura 2 mostra o percentual de sujeitos que responderam corretamente de zero a três atividades. Observamos que mais da metade dos alunos das $4^{\mathrm{a}}$ e $5^{\mathrm{a}}$ séries e, ainda, dos alunos iniciantes do curso Pedagogia não acertaram nenhuma das três atividades. Entretanto, nota-se que esse índice sofre melhora no que diz respeito aos alunos concluintes e, mais ainda, aos professores, embora, entre os participantes desse último grupo se observe um percentual de $16,3 \%$ de professores que não acertaram qualquer uma das três questões. Um outro dado que salta aos olhos é a constatação de que nenhum dos grupos de alunos (tanto do Ensino fundamental, quanto do Ensino superior (grupos 1, 2, 3 e 4) conseguiu um percentual de sucesso nas três questões superior a 3,3\%. De fato, o grupo dos professores diferenciou-se dos demais, apresentando um desempenho significativamente superior, apesar de apenas 18,6\% terem conseguido acertar as três questões.

FIGURA 2 - PERCENTUAL DOS SUJEITOS SEGUNDO O NÚMERO DE RESPOSTAS CORRETAS POR GRUPO

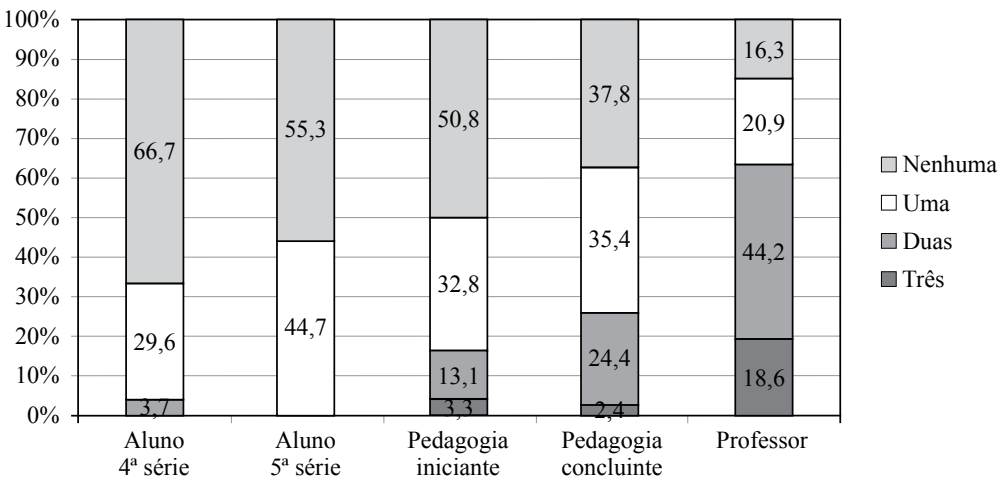


Analisando o desempenho dos grupos, medido por meio do número médio de respostas, essas diferenças foram estatisticamente significativas $(F(4,282)=$ $20,552 ; p=0,000)$. No entanto, o único grupo que apresentou um desempenho claramente superior aos demais foi o dos professores, conforme o teste de Tukey apresentado na Tabela 1 a seguir.

TABELA 1 - DESEMPENHO DOS ALUNOS NAS TRÊS ATIVIDADES POR GRUPOS

\begin{tabular}{l|c|c|c}
\hline Grupos & No de sujeitos $^{\mathbf{2}}$ & Média $\left(^{*}\right)$ & Desvio padrão \\
\hline Aluno $4^{\mathrm{a}}$ & 54 & $0,37 \mathrm{a}$ & 0,56 \\
\hline Aluno $5^{\mathrm{a}}$ & 47 & $0,45 \mathrm{ab}$ & 0,50 \\
\hline Pedagogia iniciante & 61 & $0,69 \mathrm{bc}$ & 0,83 \\
\hline Pedagogia concluinte & 82 & $0,91 \mathrm{c}$ & 0,85 \\
\hline Professor & 43 & $1,65 \mathrm{~d}$ & 0,97 \\
\hline Total & $\mathbf{2 8 7}$ & $\mathbf{0 , 8 0}$ & $\mathbf{0 , 8 7}$ \\
\hline
\end{tabular}

${ }^{*}$ ) Médias com letras iguais não diferem estatisticamente segundo o teste de Tukey.

Portanto, considerando as questões de média em geral, nota-se que ela está longe de ser algo trivial para qualquer um dos cinco grupos. Interessa-nos, contudo, proceder com a análise qualitativa dos resultados, considerando os desempenhos dos grupos em cada uma das questões. Dessa forma poderemos investigar o grau de dificuldade de cada uma das questões isoladamente e, assim, buscar entender o que na média traz maior dificuldade para o bom desempenho na questão.

\section{Análise qualitativa dos resultados}

A Figura 2 mostra o desempenho dos grupos por atividade. Podemos observar que os alunos das $4^{\mathrm{a}}$ e $5^{\mathrm{a}}$ séries tiveram desempenho próximo e não superaram $25,5 \%$ de acerto em nenhuma das atividades; os alunos do curso de Pedagogia (iniciantes e concluintes) apresentaram um desempenho ligeiramente superior aos alunos das séries iniciais do ensino fundamental, porém abaixo de $41,5 \%$ e os professores apresentaram um desempenho superior, principalmente na Atividade 2 (74,4\%), porém ainda aquém do esperado, uma vez que esses, além de terem curso superior, utilizam frequentemente o conceito de média na sua prática profissional. 
FIGURA 3 - PORCENTAGEM DE ACERTO EM CADA UMA DAS ATIVIDADES, POR GRUPO

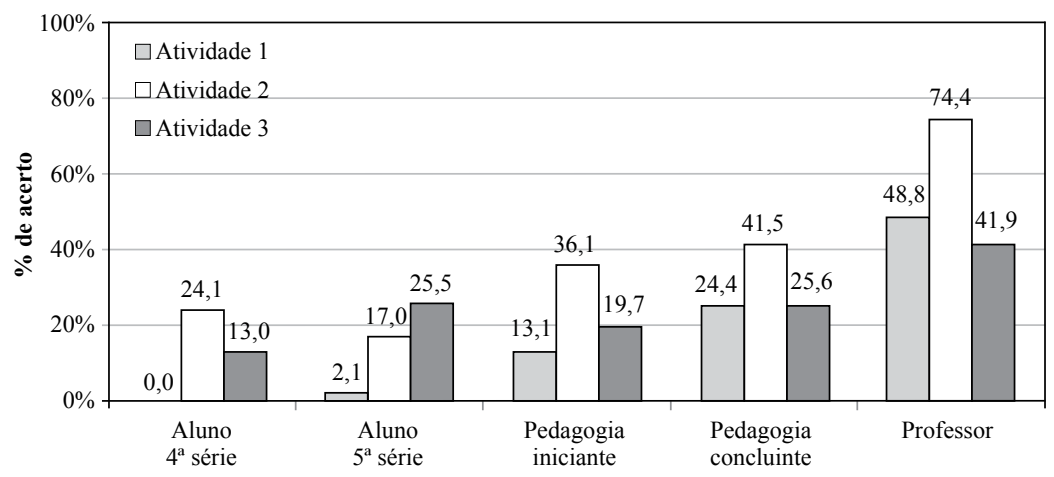

Ao analisar o desempenho nas atividades, podemos verificar que a Atividade 1 foi a que os alunos de todos os quatro grupos encontraram maior dificuldade, em particular os das $4^{\mathrm{a}}$ e $5^{\mathrm{a}}$ séries. A Atividade 2, por outro lado, foi a que apresentou menor dificuldade, com exceção do grupo 2 ( $5^{\mathrm{a}}$ série).

A Atividade 1, apresentada na figura 1, buscou identificar quais as lacunas, na compreensão de médias, os sujeitos dos diferentes grupos apresentavam no que tange às propriedades da média. Para tanto, foram analisadas as opções marcadas pelos sujeitos nos itens, quanto a verdadeiro ou falso (ver tabela 2). Para a análise foram consideradas as propriedades enunciadas por Strauss e Bichler (1988). A primeira delas é a de considerar a média como a soma dos valores da variável $(1,1,1)$. De acordo com as opções assinaladas pelos sujeitos, foi possível constatar que esta concepção, estatisticamente inválida, foi a mais frequente entre os alunos da $4^{\text {a }}$ série. Ela, porém, foi diminuindo nas séries subsequentes até atingir um percentual de 1,2\% entre os alunos concluintes e desapareceu no grupo dos professores.

Outra concepção errônea que pudemos detectar ainda na atividade 1 e a partir das respostas dos sujeitos, foi a de entender que a média só pode resultar de valores constantes e iguais a ela, isto é, o sujeito assinala apenas a alternativa $(3,3,3)$ como correta, ignorando as outras duas com valores repetidos. Esta também só apareceu nos grupos de alunos e, igualmente, apresentou trajetória decrescente com o avanço da escolaridade.

A ausência de variabilidade também pode ser uma outra concepção sem validade estatística, principalmente para os alunos iniciantes de Pedagogia $(13,1 \%)$, que assinalaram as três alternativas cujos valores eram constantes $(1,1,1 ; 3,3,3$ e 9,9,9). Em contraste, identificamos uma outra concepção oposta 
à anterior, ou seja, os valores não podem ser constantes $(1,3,6$ e 1,3,5 e 1,2,6), encontrada em $14,9 \%$ dos alunos da $5^{\text {a }}$ série. Esses alunos ( $5^{\mathrm{a}}$ série) parecem acreditar que os valores da variável não podem superar o valor da média, uma vez que $23,4 \%$ assinalaram as alternativas $(1,1,1)$ e $(3,3,3)$. No grupo dos alunos concluintes de Pedagogia foi mais difícil detectar concepções, pois $19,0 \%$ não responderam. Apareceram ainda duas outras concepções alternativas - considerar a média como múltiplo de três e considerar que um dos valores da variável tem que coincidir com a média - mas essas não superaram a casa do $10,0 \%$.

TABELA 2 - CONCEPÇÕES ALTERNATIVAS EM RELAÇÃO À MÉDIA SEGUNDO O GRUPO DE SUJEITOS

\begin{tabular}{|c|c|c|c|c|c|c|}
\hline Concepções alternativas & 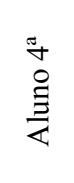 & $\begin{array}{l}\text { in } \\
\stackrel{\bigcirc}{\Xi} \\
\end{array}$ & 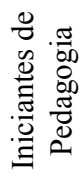 & 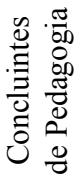 & $\begin{array}{l}\dot{0} \\
\dot{\infty} \\
\stackrel{0}{0} \\
\stackrel{0}{0}\end{array}$ & 吾 \\
\hline A média como soma $(1,1,1)$ & 25,9 & 10,6 & 4,9 & 1,2 & 0,0 & 8,0 \\
\hline Marca apenas $(3,3,3)$ & 18,5 & 10,6 & 11,5 & 7,3 & 0,0 & 9,8 \\
\hline $\begin{array}{l}\text { A média como constante } \\
(1,1,1 \text { e } 3,3,3 \text { e } 9,9,9)\end{array}$ & 5,6 & 0,0 & 13,1 & 1,2 & 4,7 & 4,9 \\
\hline $\begin{array}{l}\text { A média não pode ser constante } \\
(1,3,6 \text { e } 1,3,5 \text { e } 1,2,6)\end{array}$ & 0,0 & 14,9 & 6,6 & 3,7 & 0,0 & 4,9 \\
\hline $\begin{array}{l}\text { Os valores não superam a média } \\
(1,1,1 \text { e } 3,3,3)\end{array}$ & 0,0 & 23,4 & 4,9 & 1,2 & 0,0 & 5,2 \\
\hline Múltiplos de $3(3,3,3$ e 9,9,9) & 3,7 & 2,1 & 1,6 & 7,3 & 2,3 & 3,8 \\
\hline $\begin{array}{l}\text { Um dos números tem que ser o valor } \\
\text { da média }(3,3,3 \text { e } 1,3,6 \text { e } 1,3,5)\end{array}$ & 1,9 & 4,3 & 9,8 & 4,9 & 4,7 & 5,2 \\
\hline Em branco & 7,4 & 2,1 & 9,8 & 19,5 & 4,7 & 10,1 \\
\hline
\end{tabular}

As Figuras 3 e 4 mostram os resultados da análise qualitativa das atividades. Uma vez que observamos que as concepções mais frequentes eram a média como soma dos valores ou como ponto máximo desses valores, resolvemos analisar essas concepções entre as atividades. Observa-se que apenas os 
alunos da $4^{a}$ série demonstram considerar a média como soma para as três atividades, para os demais grupos existe uma variação grande quando comparadas as três atividades, sendo a maior diferença para os alunos da $5^{\mathrm{a}}$ série. Ressalta-se, ainda, que o grupo de professores que não apresentaram esse tipo de concepções na atividade 1 apresentaram nas atividades 2 e 3, como mostra a Figura 3.

FIGURA 3 - A MÉDIA COMO SOMA

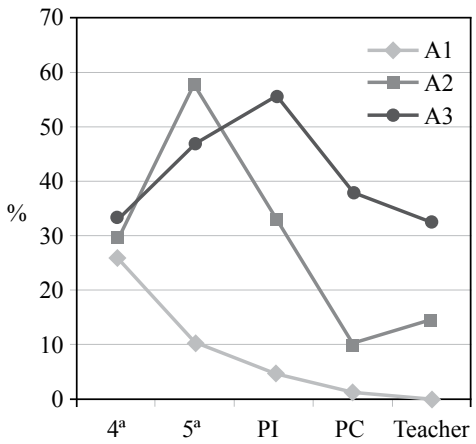

FIGURA4-AMÉDIACOMOPONTOMÁXIMO

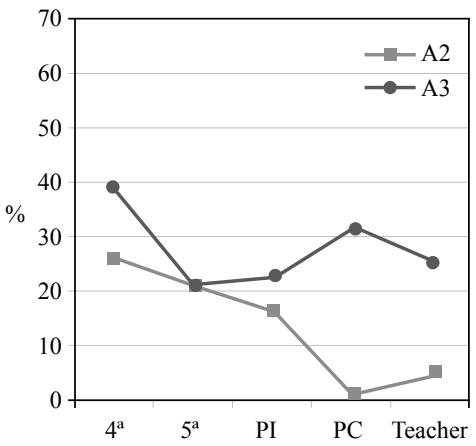

A Figura 4 também nos mostra como diferentes representações implicam em diferentes compreensões de um mesmo conceito, pois encontramos diferentes percentuais de sujeitos que demonstram compreender que a média é o ponto máximo para todos os grupos por nós investigados. Considerando as três atividades, os sujeitos se saíram melhor na segunda, a qual apresentava os dados num gráfico de barras e solicitava o cálculo da média, oferecendo três possibilidades de resposta. É possível que a representação visual tenha possibilitado uma interpretação geométrica da média aritmética e, dessa forma, o gráfico parece ter ajudado na compreensão da média.

\section{Considerações finais}

A análise dos dados revela que, em geral, a média se apresentou como um conceito difícil de ser compreendido. Observamos uma maior apropriação desse conceito em função da escolaridade, mas encontramos que professores em pleno exercício ainda apresentam concepções, sem validade estatística. 
Dentre as concepções deste tipo, encontramos um maior número de sujeitos confundindo a média com a soma dos valores, o que reforça outros estudos (POLLATSEK et al., 1981; BATANERO, et al., 1994, CAZORLA, 2003). Porém, o que nos chama atenção é que essa incompreensão ocorreu tanto entre as crianças, quanto entre os alunos do curso de graduação (futuros professores). Outra concepção comum foi confundir a média com o valor máximo dos dados, o que parece estar relacionado à falta de compreensão da propriedade da média, salientada por Strauss e Bichler (1988) de que a média só pode tomar valores entre os extremos, o que também foi detectado, principalmente, entre os alunos mais jovens. Por fim, outra concepção foi que vários sujeitos mostraram acreditar que a média tem que coincidir com pelo menos um dos valores. Estes resultados vêm ao encontro de outros estudos sobre a média já referidos anteriormente.

Estes resultados mostram o quanto ainda é preciso investir em processos de formação de professores, principalmente na formação inicial. É preciso também tornar acessível os resultados das pesquisas para os professores, a fim de que esses encontrem subsídios para sua prática profissional e possam desenvolver um processo ensino-aprendizagem que leve, de fato, os alunos a compreenderem o conceito de média e, assim, poderem interpretar e avaliar criticamente informações estatísticas.

\section{REFERÊNCIAS}

BATANERO, C. Dificultades de los estudiantes en los conceptos estadísticos elementales: el caso de las medidas de posición central. In: LOUREIRO, C.; OLIVEIRA, F.; BRUNHEIRA, L. (Eds.). Ensino e aprendizagem da estatística. Sociedade Portuguesa de Estatística, 2000.

BATANERO, C.; GODINO, J. D.; GREEN, D. R.; HOLMES P.; EVALLECILLOS, A. Errors and difficulties in understanding elementary statistical concepts. Internation Journal of Mathematics Education in Science and Technology, v. 25, n.4, p. 527-547, 1994.

BRASIL. Ministério da Educação. Secretaria de Educação Fundamental. Parâmetros Curriculares Nacionais: matemática. Brasília: Ministério da Educação/Secretaria de Educação Fundamental. 1997. 
BRASIL. Ministério da Educação. Secretaria de Educação Fundamental. Parâmetros Curriculares Nacionais: matemática. Brasília: Ministério da Educação/Secretaria de Educação Fundamental, 1998.

BRASIL. Ministério da Educação. Secretaria de Educação Média e Tecnológica. PCN + Ensino Médio: orientações educacionais complementares aos Parâmetros Curriculares Nacionais - Ciências da Natureza, Matemática e suas Tecnologias. Brasília: Ministério da Educação, 2002.

BRASIL. Ministério da Educação. Secretaria de Educação Média e Tecnológica. Orientações curriculares nacionais para o Ensino Médio - Ciências da Natureza, Matemática e suas Tecnologias. Brasília: Ministério da Educação, 2006.

CAZORLA, I. M. Média aritmética: um conceito prosaico e complexo. Anais do IX Seminário de Estatística Aplicada, Rio de Janeiro, 2003.

GAL, I. Adult's Statistical Literacy: meanings, components, responsibilities. International Statistical Review, v. 70, n.1, p. 1-25, 2002.

POLLATSEK, A.; LIMA, S. E.; WELL, A. D. Concept or computation: students' understanding of the mean. Educational Studies in Mathematics, 12, p. 191-204, 1981.

SELVA, A.; BORBA, R. O uso de diferentes representações na resolução de problemas de divisão inexata. Anais da $28^{a}$ Reunião Anual da Associação Nacional de Pós-Graduação e Pesquisa em Educação - GT19, 2005.

STRAUSS, S.; BICHLER, E. The development of children's concepts of the arithmetic average. Journal for Research in Mathematics Education, v.19, n.1, p. 64-80, 1988.

WATSON, J. What's the point? The Australian Mathematics Teacher, v. 52, n. 2, p. 40-43, 1996.

WATSON, J.; CALLINGHAM, R. (2003). Statistical Literacy: a complex hierarchical construct. Statistics Education Research Journal, v. 2, n. 2, p. 3-46, 2003. Disponível em: <http://fehps.une.edu.au/serj>. Acesso em: 20/10/2008.

Texto recebido em 08 de abril de 2009.

Texto aprovado em 06 de abril de 2010. 